\section{O papel das Classificações da OMS - CID e CIF nas definições de deficiência e incapacidade}

\section{The role of WHO Classifications - ICD and ICF - on definitions of disability}

\section{Heloisa Brunow Ventura Di Nubila}

\section{Cassia Maria Buchalla}

Centro Colaborador da OMS para a Família de Classificações Internacionais em Português, Departamento de Epidemiologia da Faculdade de Saúde Pública Universidade de São Paulo

Correspondência: Heloisa Brunow Ventura Di Nubila. Av. Dr. Arnaldo, 715 - sala 40 subsolo (CBCD). CEP 01246-904 São Paulo, SP. E-mail: hdinubil@usp.br

\section{Resumo}

A Organização Mundial de Saúde tem hoje duas classificações de referência para a descrição dos estados de saúde: a Classificação Estatística Internacional de Doenças e Problemas Relacionados à Saúde, que corresponde à décima revisão da Classificação Internacional de Doenças (CID-10) e a Classificação Internacional de Funcionalidade, Incapacidade e Saúde (CIF). A utilização da CIF vem sendo aguardada com grande expectativa pelas organizações de pessoas com deficiências e instituições relacionadas. A falta de definição clara de "deficiência" ou "incapacidade" tem sido apontada como um impedimento para a promoção de saúde de pessoas com deficiência. É importante que essas definições, especialmente no âmbito legislativo e regulamentar, sejam consistentes e se fundamentem num modelo coerente sobre o processo que origina as situações de incapacidade. Este artigo tem como objetivo apresentar elementos da CID-10 e da CIF, e o papel que desempenham para definir deficiência e incapacidade. Os componentes da CIF podem contribuir para diferentes campos de aplicabilidade no que diz respeito ao entendimento das definições de deficiência ou incapacidade a partir do conceito de funcionalidade e dos fatores contextuais.

Palavras-chave: Classificação. CID-10. CIF. Deficiências. Incapacidade. 


\section{Abstract}

The World Health Organization has two current reference classifications for the description of health conditions: ICD-10 (International Statistical Classification of Diseases and Health Related Problems) and ICF (International Classification of Functioning, Disability and Health). The utilization of the ICF is being awaited with high expectations by organizations of people with disabilities and related institutions. The lack of a clear definition of "disability" is being pointed out as a deterrent for promoting the health of people with disabilities. It is important that these definitions, especially for legal and regulatory fields, be consistent and based on a coherent model on the process that leads to situations of disability. This article aims to present elements of the ICD-10 and ICF and their role on definitions of disability. The ICF components could contribute to different fields of applicability regarding the understanding of definitions of disability through functioning and contextual factor concepts.

Kewwords: Classification. IDC-10. ICF. Deficiencies. Incapacity.

\section{Introdução}

A definição e a mensuração da incapacidade tornaram-se tema de crescente interesse, em especial a partir do momento em que as pessoas começaram a viver mais tempo, quando aumentaram as doenças crônicas e suas conseqüências ${ }^{1}$. A falta de uma definição clara de "deficiência ou incapacidade" tem sido apresentada como um impedimento para a promoção da saúde de pessoas com deficiência. A vigilância e a intervenção dependeriam da capacidade para identificar as pessoas que deveriam ser incluídas nesta definição ${ }^{2}$.

Os termos "queixas médicas, moléstia, enfermidade, doença crônica, distúrbio, limitações funcionais, deficiência e incapacidade para o trabalho" representam fenômenos complexos e mal definidos. A deficiência muitas vezes não pode ser observada diretamente, mas pode ser inferida a partir de causas presumidas (prejuízos, danos) com suas distintas conseqüências, isto é, uma restrição ou incapacidade para desempenhar normalmente vários papéis, principalmente de trabalho. Danos à saúde que causam deficiência precisam ser avaliados medicamente, mas a certificação clínica de dano, embora seja necessária, pode não ser suficiente para atestar a incapacidade para o trabalho ou elegibilidade para benefícios ${ }^{3}$. O processo de certificação de deficiência ou incapacidade pode às vezes ser bastante litigioso devido a diferenças entre suas definições legais, administrativas, sociais e culturais. Diferentes sistemas definem deficiência ou incapacidade de acordo com suas próprias necessidades e regulações, mas as definições em geral carecem de critérios específicos, impossibilitando determinações mais precisas ${ }^{4}$. Para quem certifica a condição, para fins de acesso a vários tipos de benefícios, a questão das definições de deficiência apresenta-se de modo constante. Quem é e quem não é elegível? Ao utilizar habitualmente os códigos da CID-10 para cobrir exigências legais, os médicos e outros profissionais podem questionar quais são os limites, dentro de um conjunto de 
situações muito vasto e heterogêneo. Estes profissionais podem ainda questionar o uso de definições legais vagas como parâmetro, estas fortemente ancoradas em diagnósticos médicos, com pouquíssima orientação quanto a aspectos funcionais.

As definições de incapacidade de âmbito legislativo e regulamentar têm de ser consistentes e se fundamentar em um modelo coerente sobre o processo que origina a incapacidade, para que o desenvolvimento das políticas seja baseado em dados válidos e fiáveis sobre o estado funcional da população ${ }^{5}$. A publicação da CIF neste caminho, como classificação complementar à CID, com seu foco na funcionalidade, trouxe o interesse em explorar as sobreposições e interfaces das duas classificações, no que tange aos próprios limites a serem desenhados para as definições de deficiência. Associar as categorias de diagnósticos de estados de saúde da CID-10 com os elementos da recém-apresentada CIF, oferecendo uma discussão sobre a prática possível a partir das duas classificações, pode contribuir para um melhor entendimento de possíveis definições de deficiência ou incapacidade.

Os autores, através de documentação assinada e enviada a RBE, declaram não existir nenhum tipo de conflito de interesses.

\section{Breve histórico das duas classificações}

A Classificação Internacional de Doenças (CID) veio sendo estruturada, por mais de um século, primeiro como forma de responder à necessidade de conhecer as causas de morte. Passou a ser alvo de crescente interesse e seu uso foi ampliado para codificar situações de pacientes hospitalizados, depois consultas de ambulatório e atenção primária, sendo seu uso sedimentado também para morbidade. A sua Décima Revisão, denominada "Classificação Estatística Internacional de Doenças e Problemas Relacionados à Saúde", ou de forma abreviada “CID-10", é a mais recente revisão da “Classificação de Bertillon” de $1893^{6}$, que era inicialmente uma classificação de causas de morte, e apenas a partir da Sexta Revisão passou a ser uma classificação que incluiu todas as doenças e motivos de consultas, possibilitando seu uso em morbidade ${ }^{7}$.

O conceito de uma "Família" de Classificações foi surgindo na medida da percepção dos usuários de que uma classificação de doenças não seria suficiente para todas as questões relacionadas à saúde. Segundo esse conceito, a CID atenderia as necessidades de informação diagnóstica para finalidades gerais, enquanto outras classificações seriam usadas em conjunto com ela, tratando com diferentes enfoques informações sobre procedimentos médicos e cirúrgicos e as incapacidades, entre outros. Assim, a partir da Décima Revisão foi aprovada a idéia de desenvolver uma "família" de classificações para os mais diversos usos em administração de serviços de saúde e epidemiologia ${ }^{6,7}$.

A idéia de desenvolvimento da CIF partiu da necessidade de cobrir as questões que não eram alcançadas pela CID, a princípio as conseqüências das doenças. Isto foi amplamente publicado, avaliado e revisado. Assim, a CIF pertence à "família" apresentada acima, e foi desenvolvida a partir da "Classificação Internacional das Deficiências, Incapacidades e Desvantagens" publicada em 1980 (nesta versão, Classificação Internacional de Deficiências, Incapacidades e Desvantagens ou International Classification of Impairments, Disabilities and Handicaps, conhecidas pelas siglas CIDID em português ou ICIDH do nome em inglês), em caráter experimental para propósitos de teste, que incorporava categorias que correspondiam às conseqüências duradouras das doenças ${ }^{9,10}$.

A CIF foi desenvolvida após estudos de campo sistemáticos e consulta internacional que começaram no início dos anos 90, sendo então aprovada em maio de 2001 para uso internacional ${ }^{8,11}$. Foi endossada como segunda edição da ICIDH ${ }^{11}$, refletindo o conhecimento e o pensamento de uma década diferente ${ }^{12,13}$. Organizações como a Rehabilitation International (RI) tiveram participação importante em questões conceituais ao longo das revisões sucessivas da CIDID/ICIDH até a versão final da CIF . 
Foi um processo com ampla participação internacional, envolvendo mais de 50 países e 1.800 peritos com todos os centros colaboradores, grupos de trabalho específicos para algumas partes, instituições internacionais representativas e redes internacionais ${ }^{11,13}$.

A versão final da Classificação Internacional de Funcionalidade, Incapacidade e Saúde, foi disponibilizada nos seis idiomas oficiais na página da família de classificações da OMS e publicada na língua portuguesa para todos os países lusófonos em novembro de 2003 também disponível na página http://www.fsp.usp.br/ $\operatorname{cbcd}^{11,13,14}$.

\section{CID-10 e CIF}

A OMS agora tem duas classificações de referência para a descrição dos estados de saúde: a CID-10 e a CIF ${ }^{12}$. Na família de classificações internacionais da OMS, as condições ou estados de saúde propriamente ditos (doenças, distúrbios, lesões, etc.) são classificados principalmente na CID-10, que fornece um modelo basicamente etiológico, embora tenha uma estrutura com diferentes eixos ou grandes linhas de construção, entre estes o etiológico, o anátomo-funcional, o anátomo-patológico, o clínico e o epidemiológico. A funcionalidade e incapacidade associadas aos estados de saúde são classificadas na $\mathrm{CIF}^{8,11}$.

Uma classificação de doenças é definida como um sistema de categorias atribuídas a entidades mórbidas segundo algum critério estabelecido, com vários eixos possíveis de classificação. Um determinado eixo pode ser selecionado, dependendo do uso das estatísticas elaboradas. Em uma classificação estatística de doenças, todas as entidades mórbidas devem estar incluídas dentro de um número administrável de categorias ${ }^{6}$.

Como a CID-10 é uma publicação oficial da OMS, os países membros devem adotá-la para finalidade de apresentações estatísticas das causas de morte (mortalidade) ou das doenças que levam a internações hospitalares ou atendimentos ambulatoriais (morbidade). Hoje é a classificação diagnóstica padrão internacional para propósitos epidemiológicos gerais e administrativos da saúde, incluindo análise de situação geral de saúde de grupos populacionais e o monitoramento da incidência e prevalência de doenças e outros problemas de saúde. Embora a CID seja adequada para estas aplicações, ela nem sempre permite a inclusão de detalhes suficientes para algumas especialidades, e às vezes pode ser necessária a informação acerca de diferentes atributos das afecções classificadas ${ }^{6}$. A CID registra uma condição anormal de saúde e suas causas, sem registrar o impacto destas condições na vida da pessoa ou paciente, e é hoje uma exigência legal para todos os benefícios e atestados relacionados ao paciente $\mathrm{e}^{15}$. A maioria das leis no Brasil, que concede benefícios a pessoas com deficiência, tem como exigência a apresentação de laudo médico, algumas vezes acompanhado da avaliação e assinatura de outros profissionais de equipes multiprofissionais, com o preenchimento de campos específicos para códigos da CID ou a simples informação destes códigos em atestados médicos em receituário comum assinado por médico.

A CIF, como uma classificação que se propõe a retratar os aspectos de funcionalidade, incapacidade e saúde das pessoas, o que pode ser entendido como um objetivo geral, adquire um caráter multidisciplinar, com possibilidade de aplicação em todas as culturas e trazendo pela primeira vez a incorporação dos aspectos de contexto. Isto a torna um instrumento bem mais complexo que a CID, o que faz com que exija um maior detalhamento. Entre seus objetivos específicos, está o de oferecer um modelo para a compreensão dos estados de saúde e de condições relacionadas, bem como de seus determinantes e efeitos, além de estabelecer uma linguagem comum para a descrição completa da experiência de saúde de um indivíduo, melhorando a comunicação entre as pessoas interessadas e os profissionais da área. Como instrumento estatístico, a CIF pode servir para a apresentação e comparação de dados entre países, disciplinas de cuidados de saúde, entre diferentes tipos de serviços e longitudinalmente no tempo ${ }^{8,11}$. 
Estes objetivos estão interrelacionados e envolvem a necessidade de traduzir um modelo teórico em um sistema prático e útil para aplicação em diferentes usos, especialmente como base para estatística.

A CIF é uma classificação de saúde e de domínios relacionados à saúde e estes são agrupados de acordo com suas características comuns (tais como origem, tipo ou similaridade) e ordenados de um modo significativo ${ }^{8}$. Com uma estrutura que obedece a um modelo, sua informação está organizada em três componentes:

- O "Corpo", compreendendo duas classificações, uma para funções do corpo e uma para estruturas do corpo. Os códigos usados para funções corporais são precedidos da letra "b" (de body functions) e as estruturas corporais pela letra "s" (de structure);

- "Atividade" e "Participação", que é o que o "corpo" realiza. Representam aspectos da funcionalidade a partir da perspectiva individual e social, incluídas em uma lista única que engloba todas as áreas vitais, das quais fazem parte desde a aprendizagem básica até interações interpessoais ou de trabalho ${ }^{8,9}$. Os códigos para "atividades e participação são precedidos pela letra "d" (de domain).

- O "contexto", que é a circunstância em que o "corpo" realiza suas "atividades e participação". Entre os fatores contextuais estão incluídos os "fatores ambientais”, que representam o ambiente físico, social e de atitudes nos quais as pessoas vivem e conduzem suas vidas $\mathrm{e}$ que têm um impacto sobre todos os três componentes. Estes são organizados em uma lista partindo do ambiente mais próximo do indivíduo para o ambiente mais geral e são representados pelos códigos que se iniciam com a letra "e" (de environment).

As definições operacionais da CIF descrevem os atributos essenciais de cada componente e contêm pontos de ancoramento para avaliação, de modo que estas podem ser traduzidas em questionários ou, ao contrário, os resultados obtidos com o uso de instrumentos de avaliação podem ser codificados usando-se os termos da CIF. Por exemplo, entre as funções do corpo, a "visão" é definida como a possibilidade de uma pessoa ver objetos com nitidez a distâncias variadas, além do campo visual e da qualidade da visão, enquanto a gravidade da dificuldade de visão pode ser codificada como de nível leve, moderado, grave ou completo $^{8,11}$.

O uso de qualquer código da CIF deve ser acompanhado por pelo menos um qualificador, que dá a medida da gravidade do problema em questão, como apontado acima (leve, moderado, grave ou total). O qualificador é apresentado como mais um dígito adicionado ao código e completa a informação fornecida. Sem os qualificadores, os códigos não têm significado quando usados para avaliar a situação de saúde de indivíduos ou em estudos de casos $^{8,11}$. Assim, ao avaliar as condições das pessoas com problemas, deficiências, doenças, quando estas interferem (ou não) na execução de atividades, os qualificadores permitem mensurar, tanto a interferência negativa, gerando uma limitação, como a positiva, melhorando a execução destas atividades. Essa avaliação pode ainda considerar o que é possível fazer em um ambiente padrão, de teste, e na vida real.

Os domínios de Atividade e Participação são qualificados por dois qualificadores: desempenho e capacidade. O qualificador de desempenho descreve "o que um indivíduo faz no seu ambiente real". Este "ambiente real" representa o contexto social e físico em que o indivíduo vive no seu dia-a-dia. Assim, o qualificador "desempenho" pode também ser entendido como "envolvimento em uma situação de vida" ou "a experiência vivida" da pessoa no contexto real em que vive. O qualificador de capacidade descreve "a capacidade de um indivíduo para executar uma tarefa ou ação em um ambiente uniforme" e seria o nível provável mais elevado que uma pessoa poderia alcançar na execução de uma determinada tarefa ou ação em um dado momento. Este "ambiente uniforme” (ou neutro) representa os 
ambientes relevantes para a tarefa ou ação especificada, e não deve ter barreiras ou obstáculos, de modo a permitir a avaliação da total capacidade do indivíduo ${ }^{8,11}$.

Os fatores ambientais, que são externos ao indivíduo, podem ter influência positiva ou negativa sobre a sua participação como um membro da sociedade, no desempenho de atividades ou mesmo sobre alguma função ou estrutura corporal ${ }^{8,11}$.

Os fatores pessoais, que ainda não são detalhados na CIF, são as características individuais de cada pessoa que não são parte de uma condição de saúde ou estado de saúde, mas influem na maneira como o indivíduo lida com a doença e suas conseqüências. Incluem raça, gênero, idade, nível educacional, experiências, estilo de vida, aptidões, outras condições de saúde, preparo físico, hábitos, modos de enfrentamento, condição ou nível social, profissão e mesmo a experiência passada e atual ${ }^{8,11}$.

A informação sobre a doença e as habilidades funcionais afetadas está incluída nas descrições de estados de saúde. A CIF oferece um perfil ou resumo destas habilidades funcionais, que são definidas de um modo padronizado para permitir de alguma maneira observação e mensuração. As descrições de saúde, partindo do modelo da CIF, podem oferecer uma ferramenta útil para chegar a exercícios de avaliação conceitualmente compreensíveis e significantes. Usando o modelo da CIF, pode-se estudar a mudança (adaptação, enfrentamento, ajustamento e acomodação) após um evento que produz uma determinada condição de saúde, uma vez que a CIF permite a codificação e o uso de qualificadores para medidas de capacidade, fatores ambientais e fatores pessoais ${ }^{8,11}$.

Em suma, a CID-10 e a CIF são consideradas classificações complementares e os usuários são estimulados a utilizá-las em conjunto. A CID-10 fornece um "diagnóstico" de doenças, distúrbios ou outras condições de saúde e essas informações são complementadas pelas informações adicionais fornecidas pela CIF sobre funcionalidade. Em conjunto, as informações sobre o diagnóstico e sobre a funcionalidade fornecem uma imagem mais ampla e mais significativa para descrever a saúde das pessoas ou de populações, que pode ser utilizada, entre outros, para propósitos de tomada de decisão $0^{8,11}$. Enquanto a CID-10 fornece os códigos para mortalidade e morbidade, a CIF fornece os códigos para descrever a variação completa de estados funcionais que capturam a experiência completa de saúde ${ }^{16}$. O modelo mais amplo de funcionalidade, incapacidade e saúde oferecido pela CID-10 e pela CIF, quando aplicado para a construção de instrumentos para levantamentos de saúde e incapacidade, poderia reforçar o campo da pesquisa em saúde e incapacidade, na infância ${ }^{17}$, mas também nas outras etapas do ciclo de vida.

\section{Definições de deficiência ou incapacidade da CIF}

O desenvolvimento de uma terminologia formal relacionada à funcionalidade e à incapacidade constitui um desafio, especialmente devido à ambigüidade conceitual dentro deste campo. A CIF é uma grande fonte de termos relevantes, conceitos e relações necessárias para o desenvolvimento de terminologias formais, e assim oferece um importante ponto de partida neste desafio ${ }^{18}$.

Durante décadas foram disponibilizadas ferramentas úteis para coletar dados sobre causas de morte e modos de estimar a mortalidade da população. Embora as disciplinas da reabilitação tenham produzido inúmeros instrumentos de avaliação e medidas de qualidade de vida, faltava uma completa classificação que pudesse assegurar coleta de dados confiáveis e comparabilidade internacional. Esta foi a primeira motivação que levou ao desenvolvimento da CIF, que agora serve como modelo da OMS para saúde e incapacidade, como base conceitual para a definição, medidas e formulações de política para todos os aspectos da deficiência ou incapacidade ${ }^{19,20}$.

Ao construir as definições das categorias da CIF, foram consideradas características ideais das definições operacionais. São 
consideradas características ideais das definições elas terem um significado e consistência do ponto de vista lógico, identificando unicamente o conceito mencionado pela categoria; e serem exclusivas e precisas, apresentando as características ou qualidades essenciais do conceito e obedecendo às regras de taxonomia. Os termos utilizados devem ser neutros, sem conotações negativas. Cada definição contém, como também se observa na CID, notas de inclusão com sinônimos e exemplos, bem como notas de exclusão para alertar sobre possíveis confusões com termos relacionados ${ }^{11}$.

A seguir são alistadas as definições dos componentes da CIF.

- Funções Corporais: são as funções fisiológicas ou psicológicas dos sistemas do corpo.

- Estruturas Corporais: são as partes anatômicas do corpo tais como órgãos, membros e outros componentes.

- Deficiências: são problemas na função ou estrutura corporal, tais como um desvio ou perda significativos.

- Funcionalidade: refere-se a todas as funções do corpo e desempenho de tarefas ou ações como um termo genérico.

- Incapacidade: serve como um termo genérico para deficiências, limitações de atividades e restrições à participação, com os qualificadores de capacidade ou desempenho. A CIF também alista fatores ambientais que interagem com todos estes construtos ${ }^{8}$.

Na CIF, o termo deficiência corresponde, como descrito acima, a alterações apenas no nível do corpo, enquanto o termo incapacidade seria bem mais abrangente, indicando os aspectos negativos da interação entre um indivíduo (com uma determinada condição de saúde) e seus fatores contextuais (fatores ambientais ou pessoais) ${ }^{11}$, ou seja, algo que envolva uma relação dinâmica. Um indivíduo pode apresentar uma deficiência (no nível do corpo) e não necessariamente viver qualquer tipo de incapacidade. De modo oposto, uma pessoa pode viver a incapacidade sem ter nenhuma deficiência, apenas em razão de estigma ou preconceito (barreira de atitude).

A CIF faz um deslocamento paradigmático do eixo da doença para o eixo da saúde, trazendo uma visão diferente da saúde, que permite entender a condição ou estado de saúde dentro de contextos específicos. Como classificação de saúde, a CIF introduz um novo modo de compreender a situação de saúde de indivíduos ou populações, mais dinâmico e mais complexo, compatível com o quadro multidimensional que envolve a experiência completa de saúde.

O modelo dinâmico da CIF é mostrado abaixo, na Figura 1, incluindo os fatores contextuais.

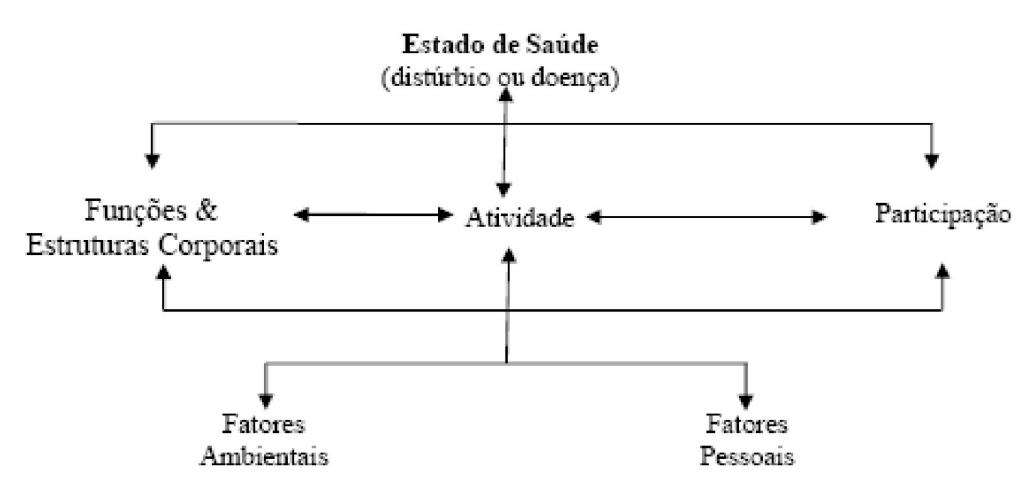

Fatores Contextuais

Fonte: OMS, CIF, 200311 / Source: WHO, ICF, 2003

Figura 1 - Interações entre os componentes da CIF

Figure 1 - Interactions between ICF components 
Uma importante característica da abordagem que foi adotada na CIF é a "universalização" do entendimento de deficiência ou incapacidade, pois reconhece a população inteira como sendo passível de apresentar uma doença crônica, deficiência ou incapacidade, como uma condição humana compartilhada ${ }^{21}$.

O esquema da CIF não fornece limites para definir quem é deficiente e quem não é; em vez disso, ela reconhece aspectos e graus de deficiência ou incapacidade ao longo de toda a população. Muitos usos estatísticos de dados de deficiência ou incapacidade não requerem o estabelecimento de limites, não existindo necessidade, nestas abordagens, de uma definição de quem se conta como deficiente e quem não. Assim, a comparação internacional de estatísticas de deficiência não requer necessariamente pontos de limite para serem resolvidos ${ }^{21}$. A CID-10 e a CIF são classificações amplas, que podem descrever qualquer estado de saúde ou de funcionalidade, sem definir limites. Na realidade, a CID-10 faz parte do modelo da CIF, estando colocada no primeiro módulo da Figura 1, que representa o seu modelo dinâmico, no lugar reservado às condições ou estados de saúde, que incluem os distúrbios ou doenças. É este modelo que se mostra como o mais aproximado da descrição da experiência de incapacidade vivida pelos indivíduos em qualquer condição de saúde, e portanto útil para apreender as variáveis envolvidas nesta situação dinâmica de interação do indivíduo com um determinado contexto.

Seus componentes descrevendo as experiências relacionadas à saúde substituem os termos "deficiência", "incapacidade" e "desvantagem", usados anteriormente, permitindo estender seus significados para incluir também, e principalmente, as experiências positivas. Os novos termos são definidos e detalhados dentro da classificação. É importante lembrar que estes são usados com significados específicos e podem diferir do seu uso corriqueiro ${ }^{8}$. Assim, é possível observar o uso intercambiável das palavras deficiência e incapacidade, tanto na língua portuguesa, como na inglesa e em outras.
Na descrição de desfechos de saúde não fatais, a CIF pode funcionar como uma " $\mathrm{Pe}$ dra de Rosetta", assim como aponta Üstun (2002), ou seja, como uma matriz de tradução para permitir a interlocução entre vários instrumentos usados para descrever ou avaliar diferentes estados de saúde. Segundo esta idéia, quase todos estes instrumentos poderiam ser mapeados dentro da CIF, que representaria um modelo comum ${ }^{11}$.

Tanto no setor da saúde como em outros setores que necessitam avaliar o estado funcional das pessoas, como é o caso da previdência social, do emprego, da educação e dos transportes, entre outros, a CIF pode desempenhar um papel importante ${ }^{5}$.

Segundo Frank Mulcahy (2005), secretário da "Disabled Peoples' International" (DPI), "por muitos anos, esta organização e muitas de outras organizações nãogovernamentais (ONGs) internacionais não adotaram uma definição de deficiência ou incapacidade. Isto se deveu a muitas circunstâncias, incluindo as seguintes, mas não restritas a estas: muitas definições diferentes usadas na legislação em diferentes países; a maioria das definições em uso eram definições médicas; havia problemas com traduções de diferentes definições; aceitação em alguns países de termos rejeitados em outros; a CIDID/ICIDH"22.

A CIDID/ICIDH, como um dos motivos, defendidos por Mulcahy, para não adotar uma definição de deficiência ou incapacidade, possivelmente se deve ao modelo médico no qual se baseava. Neste, a falta de mudança ao longo do tempo e o fluxo unidirecional da deficiência para incapacidade e para desvantagem foi alvo de muitas críticas ${ }^{11}$. A adoção de representações gráficas alternativas resultou no modelo dinâmico multidirecional e interativo da $\mathrm{CIF}^{11}$, apresentado na Figura 1.

Para Mulcahy, pode-se ter qualquer número de definições usadas para diferentes legislações, e isto cabe, particularmente, para definições de deficiência ou incapacidade usadas para Pagamentos de Benefícios em comparação com as definições Educacionais, de Treinamento e Emprego ${ }^{22}$. 
A DPI foi representada no processo de revisão da CIDID/ICIDH para se chegar ao documento final, a CIF, que adota também o modelo social de deficiência ou incapacidade, em comparação com o modelo médico previamente usado. Mulcahy ${ }^{22}$ afirma que a DPI se viu requisitada a declarar, em várias ocasiões, qual definição de deficiência ou incapacidade gostaria de ver utilizada e que a definição apresentada pelo modelo da CIF poderia ser utilizada para os seus propósitos, ou seja. "o resultado da interação entre uma pessoa com uma deficiência e as barreiras ambientais e de atitudes que possa enfrentar". Assim, Mulcahy propõe que esta poderia ser utilizada para o momento como definição preferida esperando que se possa ter uma definição melhorada quando o Conselho Mundial da DPI permitir um debate sobre esta questão ${ }^{22}$.

Esta opção pela definição da CIF, com os argumentos apresentados, possivelmente se deve ao importante apoio e participação das ONG de pessoas com deficiência na mudança do modelo da CIDID/ICIDH até o modelo atual da CIF, descrito como um modelo bio-psico-social, mais adequado para o entendimento das situações de incapacidade vividas em diferentes contextos.

Para Chaterji e col. (1999), definições de "deficiência ou incapacidade" não deveriam ser determinadas pelo modo como os dados serão usados. Elas deveriam ser determinadas por um modelo conceitual claro e coerente que se prestasse a testagem empírica. As definições poderiam ser baseadas em pontos de ancoramento e limites, não meramente aqueles que se referem a dificuldades e assistência, mas aqueles que capturam o impacto na vida do indivíduo e incorporam medidas individuais e sociais. Referindo-se ao processo de revisão que veio a resultar na CIF, o processo de medida para avaliar a situação de incapacidade é multidimensional: envolve funções e estruturas corporais, bem como as atividades de uma pessoa e o contexto ambiental ou social da pessoa cuja funcionalidade está sendo avaliada. Não existe consenso sobre que componentes deveriam ser emprega- dos no desenvolvimento de medidas para situações de incapacidade. A maioria dos instrumentos mede uma mistura de experiências que incluem sintomatologia, apoio social, carga sobre a família, satisfação, bem-estar subjetivo e qualidade de vida. O modelo da CIF deverá servir como base para futuras definições de referência para o fenômeno da incapacidade e deve constituir o padrão para medidas que possam melhor informar a política pública, a alocação de recursos, o gerenciamento de cuidados de saúde e muitos outros aspectos de uma necessária resposta social à situação de incapacidade ${ }^{1}$.

Quando a definição de deficiência precisa ser aplicada para a infância, a questão adquire uma complexidade própria. A experiência de uma condição de saúde crônica e a incapacidade para crianças e jovens não é a mesma dos adultos, em especial devido à natureza variável do desenvolvimento. Este torna particularmente difícil medir ou mesmo diagnosticar a incapacidade em crianças muito pequenas. Neste ciclo da vida, tradicionalmente, a incapacidade tem sido igualada à morbidade, e esta ambigüidade de definições faz com que os dois conceitos, de condição de saúde crônica e de deficiência ou incapacidade sejam, com freqüência, medidos e identificados de modo incorreto. Levantamentos incluindo instrumentos baseados na CID-10 e na CIF, que possam medir de modo distinto condições de saúde crônicas, os domínios de incapacidade e fatores ambientais que têm impacto sobre a incapacidade, poderiam facilitar o entendimento da incapacidade e saúde nas crianças, no contexto do modelo de saúde internacional oferecido pela OMS ${ }^{17}$.

O uso combinado da CID-10 e da CIF oferece o compromisso de uma linguagem comum para documentar a natureza e distribuição da incapacidade em crianças em sistemas de serviços de intervenção precoce ou outros serviços mais gerais para crianças $^{23}$.

Na CIF é sugerida uma lista mínima para sistemas ou pesquisas de informações de saúde ideais e mínimos, que podem ser 
pontos de partida para a exploração de categorias da CIF para definições de deficiência ou incapacidade.

\section{Comentários finais}

A CIF foi desenvolvida ao longo de duas décadas que compõem o pano de fundo de importantes mudanças de concepções e no modelo da classificação, com a aprovação e publicação de documentos de extrema relevância para o movimento de direitos das pessoas com deficiência no mundo.

A utilização da CIF pode contribuir de forma positiva para o estabelecimento de políticas públicas voltadas para as pessoas com deficiência ou incapacidade. O novo modelo trazido pela CIF, ao ser utilizado, pode fornecer informações que ajudem a estabelecer políticas de saúde, a promover a igualdade de oportunidades para todos e a apoiar a luta contra a discriminação das pessoas com deficiência ou incapacidade. A CIF foi incluída, mesmo antes da sua edição, como padrão para caracterização da deficiência na proposta do Estatuto da Pessoa com Deficiência, apresentada em consulta pública ao Senado Federal, embora esta menção tenha desaparecido e reaparecido em minutas subseqüentes. Este pode ser o reflexo da grande expectativa com a qual é aguardada, no Brasil, a utilização da CIF em português, em especial pelas organizações de pessoas com deficiências e instituições relacionadas. Outro exemplo de uso em nosso meio, mesmo reconhecendo a complexidade própria da classificação, é a aplicação da CIF em um instrumento de avaliação para a concessão do Benefício de Prestação Continuada (BPC). O Ministério do Desenvolvimento Social e Combate à Fome criou um Grupo de Trabalho Interministerial, para propor novos parâmetros e procedimentos de avaliação das pessoas com deficiência para acesso ao BPC, que optou por elaborar novos instrumentos de avaliação com base no modelo da CIF, tendo já realizado estudo piloto com grupos de médicos peritos do INSS, com boa aceitação.
A CIF, ao ser usada de uma forma mais ampla ou mais consistente, permitirá que se adote uma nova terminologia, que se padronizem conceitos e que se reflita sobre o tema, podendo contribuir para tornar mais claro o uso das palavras, para conseguir um entendimento e uma comunicação mais uniforme entre as pessoas interessadas nesta área, buscando alcançar as vantagens de sua terminologia padronizada. No Brasil, algumas leis já contêm elementos de definição da CIDID/ICIDH, mas ainda pouco se avançou para a incorporação da CIF. Algumas discussões ainda são pautadas nas definições da CIDID/ICIDH, com o uso do seu modelo linear negativo e da idéia de "handicap" ou "desvantagem". Isto possivelmente se deve ao fato de o modelo experimental da CIDID/ICIDH ter vigorado por muitos anos antes de ser revisado resultando na CIF, sendo o modelo desta ainda pouco conhecido e entendido. Isto aponta para a necessidade de que seja apresentado aos profissionais da área, grupos de pessoas com deficiência, ativistas de direitos, enfim a quantos grupos mais possam se tornar interlocutores e agentes multiplicadores com especial senso crítico, para elaborar políticas mais justas e mais claras para pessoas com deficiência ou incapacidade.

Para o futuro, espera-se o desenvolvimento de algoritmos que determinem direitos para benefícios e pensões, utilizando um melhor entendimento das definições de deficiência ou incapacidade da CIF para as questões de elegibilidade. Definições e limites desenhados a partir da classificação devem conter elementos de deficiência (no nível do corpo) associados com incapacidade (aspecto negativo de interação entre a condição de saúde e fatores contextuais), com qualificadores de gravidade.

É importante que o poder público e os formuladores de políticas participem da discussão para a obtenção de definições e avaliações mais claras e mais justas da deficiência ou incapacidade.

A CIF é de propriedade de todos os seus usuários. A sociedade interessada, em especial as pessoas com deficiência, as organiza- 
ções sociais e os formuladores de políticas, devem se apropriar do conhecimento sobre os usos potenciais da CID-10 e da CIF. O uso destas classificações como instrumentos pode contribuir para que as reais condições de vida das pessoas com deficiência venham a fazer parte das estatísticas, permitindo guiar ações e decisões, delinear políticas, definir intervenções e destinar orçamentos, entre outras.

\section{Referências}

1. Chaterji S, Üstun B, Bickenbach JE. What is disability after all? Disabil Rehabil 1999; 21: 396-8.

2. Lollar DJ. Public health and disability: emerging opportunities. Public Health Rep 2002; 117: 131-6.

3. Marin B. Transforming disability welfare policy: completing a paradigm shift. In: Prinz C, editor. European disability pension policies: eleven country trends 1970-2002. Vienna: Ashgate; 2003. p 1-54.

4. Barron BA. Disability certifications in adult workers: a practical approach. Am Fam Physician 2001; 64(9): 1579-86.

5. Portugal. I Plano de Acção para a Integração das Pessoas com Deficiências ou Incapacidade para os anos de 2006 a 2009: Resolução do Conselho de Ministros no 120/2006, de 21 de setembro. [30 ago 2007]. Disponível em: http://www.lerparaver.com/node/536

6. Organização Mundial da Saúde. Classificação Estatística Internacional de Doenças e Problemas Relacionados à Saúde: CID-10 Décima revisão. Trad. do Centro Colaborador da OMS para a Classificação de Doenças em Português. 3 ed. São Paulo: EDUSP; 1996.

7. Laurenti R. Análise da informação em saúde: 1893-1993, cem anos da Classificação Internacional de Doenças. Rev Saúde Pública 1991; 25: 407-17.

8. Üstun TB. The International Classification of Functioning, Disability and Health: a common framework for describing health states. In: Murray CJL, Solomon JA, Mathers CD, Lopez AD, editors. Summary Measures of Population Health: Concepts, Ethics, Measurement and Applications. Geneva: WHO; 2002. p. $344-8$

9. Casado D. Conceptos sobre la Discapacidad. Boletín del Real Patronato sobre Discapacidad 2001; 50: 5-13.

10. Organização Mundial da Saúde. Classificação Internacional das Deficiências, Incapacidadese Desvantagens (handicaps): um manual de classificação das conseqüências das doenças. Lisboa; 1989.
11. Organização Mundial da Saúde. CIF: Classificação Internacional de Funcionalidade, Incapacidade e Saúde. Trad. do Centro Colaborador da Organização Mundial da Saúde para a Família de Classificações Internacionais. São Paulo: EDUSP; 2003.

12. World Health Organization, Family Development Committee. Implications for the ICD of the ICF. Meeting of Heads of WHO Collaborating Centres for the Family of International Classifications. Bethesda, October; 2001 [WHO/GPE/CAS/C/01.55].

13. Egea C, Sarabia A. Classificaciones de la OMS sobre discapacidad. Boletín del Real Patronato sobre discapacidad 2001; 50:14-30.

14. International Classification of Functioning, Disability and Health Home Page http: //www3.who.int/icf/ icftemplate.cfm

15. Battistella LR, Brito CMM. Classificação Internacional de Funcionalidade (CIF) International Classification of Functioning Disability and Health (ICF). Acta Fisiatrica 2002; 9: 98-101.

16. Üstun TB, Chaterji S, Kostansjek N, Bickenbach J. WHO's ICF and functional status information in health records. Health Care Financing Review 2003; 24: 77- 88.

17. McDougall J, Miller L. Measuring chronic health condition and disability as distinct concepts in national surveys of school-aged children in Canada: a comprehensive review with recommendations based on the ICD-10 and ICF. Disabil Rehabil 2003; 25: 922-39.

18. Harris MR, Ruggieri AP, Chute CG. From clinical records to regulatory reporting: formal terminologies as foundation. Health Care Financing Review 2003; 24: 103-20.

19. McDowell I, Newell C. Measuring Health: a guide to rating scales and questionnaires. 2 ed. New York (NY): Oxford University Press; 1996.

20. Bickenbach JE. Functional status and health information in Canada: proposals and prospects. Health Care Financing Review 2003; 24: 89-102. 
21. Brunel University. Definitions of Disability in Europe: a comparative analysis: final report. September 2002. Disponível em: http://ec.europa.eu/employment_ social/publications/2004/cev502004_en.pdf. [Acessado em 30 de agosto de 2007].

22. Mulcahy F. Position paper on the Definition of Disability. May 2005. Disponível em http://v1.dpi.org/lang-en/ resources /details.php?page=74 [Acessado em $30 \mathrm{de}$ agosto de 2007].
23. Simeonsson RJ, Scarborough AA, Hebbeler KM. ICF and ICD codes provide a standard language of disability in young children. J Clin Epidemiol 2006; 59: 365-73.

Recebido em: 08/10/07 Versão final reapresentada em: 14/03/08 Aprovado em: 19/03/08 